\title{
Application of the Method of Non-Classical Approach to Certain Problems of the Theory of Anisotropic Space Plazma in Fluid Description
}

\author{
Mahammad A. Nurmammadov, Namiq S. Dzhalilov \\ Shamakhy Astrophysical Observatory of ANAS, Shamakhy, Azerbaijan \\ Email: nurmamedov_55@mail.ru,namigd@mail.ru
}

How to cite this paper: Nurmammadov, M.A. and Dzhalilov, N.S. (2021) Application of the Method of Non-Classical Approach to Certain Problems of the Theory of Anisotropic Space Plazma in Fluid Description. Journal of Applied Mathematics and Physics, 9, 1181-1193.

https://doi.org/10.4236/jamp.2021.96081

Received: April 29, 2021

Accepted: June 7, 2021

Published: June 10, 2021

Copyright $\odot 2021$ by author(s) and Scientific Research Publishing Inc. This work is licensed under the Creative Commons Attribution International License (CC BY 4.0).

http://creativecommons.org/licenses/by/4.0/

\begin{abstract}
In this paper, the new well-posed boundary value problem to the system of singular nonlinear differential equations is considered, which describes the stationary radial outflow of anisotropic plasma from the Sun (solar wind). These equations are obtained on the basis of 16-moment MHD (magnetohydrodynamic) transport equations for a collisionless magnetized plasma, which takes into account the temperature anisotropy relative to the direction of the magnetic field and the heat flux carried by the wind. This is a generalization of the classical isotropic Parker model taking into account the effects of anisotropy. In this paper, the equations under study are characterized as a nonautonomous nonlinear system of ordinary differential equations the coefficients in which degenerate and simultaneously have singularities. These equations are related to an unsolved problem in the general theory of ODEs (Ordinary Differential Equations). At first, according to the conditions of the coefficients of the equations, a non-classical boundary value problem is set, and the solvability is established for the same non-autonomous and nonlinear system of equations under consideration. The found analytical solution reconstructs numerical solutions, which are simultaneously automatically established by classical formulation of boundary value problem. Parker's solutions are also partially included in this obtained class of solutions, which is presented with strictly proves. Further, by means of the methods of " $\varepsilon$ -regularization" and "fixed point" the theorem of solvability for the considered differential equations is obtained. After constructed nonsingular system equations with well-posed boundary value problem, the analytical solutions are founded. Using the sketch of graph of these solutions their family is established.
\end{abstract}

\section{Keywords}

ODE, Non-Autonomous and Nonlinear Systems, Anisotropic MHD, Solar 
Wind Outflow, Singularity, Degenerating

\section{Introduction}

In 1957, Parker showed that although solar gravity strongly attracts the solar corona, it is still very hot at large distances, and while gravity weakens with increasing distance from the Sun, the outer coronal plasma particles supersonically escape into interstellar space [1]. Parker's model predicted that the wind should transition to supersonic flow (Mach number passes through the unit) at about 4 solar radii from the photosphere (surface). The acceleration of the wind is still not well understood and cannot be fully explained by Parker's theory. The results of many cosmic experiments and measurements of solar wind parameters near the Earth cannot be explained by the Parker model and by other theories based on the isotropic MHD [2] [3]. Since the measured parameters of the solar wind are highly anisotropic, it is necessary to develop the theory of wind modeling within the framework of the anisotropic MHD theory. MHD waves and instabilities in anisotropic plasma are considered, for example, in works [4] [5]. The theory of MHD outflow of anisotropic plasma from the Sun, taking into account the heat flux, has not been developed yet. A generalization of the Parker task in the simple case (radial stationary flow) to the case of anisotropic plasma is considered in the paper [6]. In this case, the MHD transport equations are reduced to a system of three nonlinear singular equations, which are considered in this paper. These equations are characterized as a non-autonomous nonlinear system of ordinary differential equations in which the coefficients degenerate and simultaneously have singularities. In this case, the MHD transport equations are reduced to a system of three nonlinear singular equations, which are considered in this paper. These equations are characterized as a non-autonomous nonlinear system of ordinary differential equations in which the coefficients degenerate and simultaneously have singularities. The works (see [7] [8] [9] and therein) given definition of nonclassical model problems and their equations. Namely, noted that the equation of Keldysh (see [10]) is also fit to the class of non-classical equations. Often for setting well-posed boundary value problems of the equations of mixed type (for example, in cases of degenerating, singularity, elliptical-hyperbolic equations and others) can be used from theory of M. V. Keldysh. In Section 2, considered problem as well known, consists of establishing boundary conditions corresponding to the critical point which associated with number Mach. In aerodynamically, hydro-gas dynamic problems it is important an application in the theory of M. V. Keldysh. In case of stationary, for some degenerating ordinary differential equations to applying this theory is useful. The work (see [11]) considered model equation of mixed type, which is coefficients of higher-order derivatives (with respect to $\mathrm{x}, \mathrm{t}$ ) have to Keldysh type degenerating cases. In this work our aim consists of construct boundary conditions 
for admissible $x>0$, to investigate system of ordinary differential equations and after proving solvability of this problem, finding solutions. The details of methods are given in the next sections.

\section{Problem Statement and Solvability of the Nonlinear, Non-Autonomy Systems of the Ordinary Differential Equations with Degenerated and Singular Coefficients}

Note that in the theory of ordinary differential Equations (also systems of equations) both linear and nonlinear systems of equations are quite well studied. Although, non-autonomous and nonlinear systems of ordinary equations have a number of difficulties, also the application of various special methods by appropriate formulations of boundary conditions is also solved. However, the theory of ordinary differential equations both linear and non-linear systems of equations when the coefficients degenerate, then for the solutions of such systems of equations the theory of classical ODE is almost inapplicable and refers to unsolved problems. Proceeding from these difficulties we will try to apply the theories of the so-called non-classical models and methods of non-classical equations to the system of equations considered here.

\subsection{Discussing the Solvability of Non-Classical Problem}

The considered system Equations (see [6]) being to the system of three nonlinear, non-autonomy of the ordinary equations with degenerating and singularity are:

$$
3 x^{3} \frac{\mathrm{d} X}{\mathrm{~d} x}+\frac{f_{1}(x)}{f(x)}=0,6 x^{3} \frac{\mathrm{d} Y}{\mathrm{~d} x}+\frac{f_{2}(x)}{f(x)}=0,6 x^{3} X \frac{\mathrm{d} Z}{\mathrm{~d} x}+\frac{f_{3}(x)}{f(x) A(x)}=0
$$

where, $A=\frac{Y}{X}-1, B=-\frac{9}{4} X+\bar{C}_{5}+\frac{\bar{g}}{x}-\frac{\bar{C}_{6}}{x^{2}}, \quad D=2 Z-x \bar{g}, \quad E=2 \bar{C}_{6}-x \bar{g}$,

$f=\frac{1}{2} A(A-4)+\frac{4 B}{3 X}, f_{1}=3 A D+2 E, f_{2}=A f_{1}-6 D f$,

$f_{3}=f_{1}(D-E)-12 f Z^{2}$.

The functions $X, Y, Z$ are dependent from the space variable of $x$ and $X(x)$, $Y(x), Z(x)$ which are real positives functions, but $g=1$ and the constants $C_{5}, C_{6}$ are unknowns. The functions $f=f\left(X, Y, Z ; x ; C_{5}, C_{6}\right)$;

$f_{1}=f_{1}\left(X, Y, Z ; X ; C_{5}, C_{6}\right) ; f_{2}=f_{2}\left(X, Y, Z ; X ; C_{5}, C_{6}\right) ;$

$f_{3}=f_{3}\left(X, Y, Z ; x ; C_{5}, C_{6}\right)$ are dependence from the $X, Y, Z, C_{5}, C_{6}$. However, the constants are $C_{5}, C_{6}$ unknown which must be determine by means of initial condition (in classical sense approaches). Additionally, in this system of (2.1) the coefficients $f(x)=0$ and $A(x)=0$ are including the cases of degenerating and singularity. Therefore, the boundary value problems cannot be determined directly as traditionally initial value problems for system equations of (2.1). It is impossible; but we need seek a new non-classical approaches model which allowance establishes boundary value problems of the system Equations (2.1).

Definition 2.1. Non-classical Boundary Value Problem of system (2.1). Non- 
classical approaches consists of finding the constants $C_{5}, C_{6}$ are such that in the domain $x>0$ for degenerating and singular system Equations (2.1) to establish influences the coefficients $f(x)=0$ and $A(x)=0$ such that the system becomes nonsingular systems of ODE and choosing these coefficients to find suitable solution of unknown well posed boundary value problems which is corresponding for nonlinear, no autonomy ordinary system Equations (2.1), with the degenerating and singularity coefficients. Note that, for such non-classical boundary value problems the linearization of (2.1) and for it solvability the numerical method directly using classical approaches methods are not applicable. For this reason for any $\varepsilon>0$ we can rewrite in the following form

$$
\begin{aligned}
& \left\{\begin{array}{l}
f(x) \frac{\mathrm{d} X}{\mathrm{~d} x}=-\frac{f_{1}(x)}{3 x^{3}} \\
f(x) \frac{\mathrm{d} Y}{\mathrm{~d} x}=-\frac{f_{2}(x)}{3 x^{3}} \\
f(x) A(x) \frac{\mathrm{d} Z}{\mathrm{~d} x}=-\frac{f_{3}(x)}{6 x^{3} X(x)}
\end{array}\right. \\
& \left\{\begin{array}{l}
(f(x)+\varepsilon) \frac{\mathrm{d} X}{\mathrm{~d} x}=-\frac{f_{1}(x)}{3 x^{3}} \\
(f(x)+\varepsilon) \frac{\mathrm{d} Y}{\mathrm{~d} x}=-\frac{f_{2}(x)}{3 x^{3}} \\
(f(x)+\varepsilon)(A(x)+\varepsilon) \frac{\mathrm{d} Z}{\mathrm{~d} x}=-\frac{f_{3}(x)}{6 x^{3} X(x)}
\end{array}\right.
\end{aligned}
$$

We have been using the terms supersonic and subsonic to refer to the regions by the regularizing system equations

$$
\left\{\begin{array}{l}
(f(x)+\varepsilon) \frac{\mathrm{d} U}{\mathrm{~d} x}=-F(x) \\
\frac{\mathrm{d} U}{\mathrm{~d} x}=\left(\begin{array}{l}
\frac{\mathrm{d} X}{\mathrm{~d} x} \\
\frac{\mathrm{d} Y}{\mathrm{~d} x} \\
(A+\varepsilon) \frac{\mathrm{d} Z}{\mathrm{~d} x}
\end{array}\right), F(x)=\left(\begin{array}{l}
\frac{f_{2}(x)}{3 x^{3}} \\
\frac{f_{2}(x)}{6 x^{3}} \\
\frac{f_{2}(x)}{6 x^{3} X(x)}
\end{array}\right)
\end{array}\right.
$$

Accordance to the result changing type equation from the work [11] account into stationary case $t=$ constant, if instead coefficient $\left(K_{2}(x)+\varepsilon\right)$ replacing the $(f(x)+\varepsilon)$ and making some of suitable notation, from the equation [8] formally being to the form (2.4), and it is obviously we can carry out to proof of solvability equation in [11] that, the system Equation (2.4) solvable in the space $L_{2}$. Proof of this proposition can be held identically, similarly way as it shown in the work [10] [11], in case of analogical to first order ordinary differential system equations. This proposition is formally as theoretical aspects in order to apply. On the base of this theory we need find only practice results, i.e. numerically results must be illustration in view of graphical lines. By the standard transition 
limit may be assert that the solution of regularizing system equations of (2.4) converges to the solution of system equations of (2.1) or (2.2), with suitable boundary conditions $Z\left(c^{*}\right)=c, X\left(c^{*}\right)=c, Y\left(c^{*}\right)=c \quad$ (where $c$ is the constant, will be founded from the fixed point and by the theory of fixed points these conditions is so-called as free move conditions (or unknown boundary conditions) if the constants $\mathrm{c}$ chosen as variables.

Using definition of fixed point and its properties on the critical point (which is number Mach is equal to 1, i.e. at shock point) we may determine as free initial conditions at the nearly of critical point $x=c^{*}$ with aid equalities: $B\left(c^{*}\right)=3$, $X\left(c^{*}\right)=2$. Hence, at the point $x=c^{*}$ obtain that $Y\left(c^{*}\right)=2$. In this case, taken main idea of non zero fixed point rule.

$$
\begin{aligned}
& \text { Hence, }-\frac{9}{4} \cdot 2+\bar{C}_{5}+\frac{1}{c^{*}}-\frac{\bar{C}_{6}}{\left(c^{*}\right)^{3}}=3,18 Z\left(c^{*}\right)-6 c^{*}=-2 \bar{C}_{6}+c^{*}, \\
& \bar{C}_{6}=9 Z\left(c^{*}\right)+3.5 c^{*} . \\
& \text { Thus, } \bar{C}_{5}=\frac{9 Z\left(c^{*}\right)+3.5 c^{*}}{\left(c^{*}\right)^{3}}+7.5+\frac{1}{c^{*}} . \bar{C}_{6}=9 Z\left(c^{*}\right)+3.5 c^{*} \text {. Where } Z\left(c^{*}\right) \text { is }
\end{aligned}
$$

the observation value at the point $x=c^{*}$. Hence, by the observation value of $Z\left(c^{*}\right)$ the constants are $\bar{C}_{5}, \bar{C}_{6}$ : founded $\bar{C}_{5}=C^{*}, \bar{C}_{6}=C^{* *}$ by means of known methods. Consequently, now theoriatically, acordance these constans value of $\bar{C}_{5}=C^{*}, \bar{C}_{6}=C^{* *}$ and into account of these constants in the expression of coeficints $A(x), f=f\left(X, Y, Z, x ; C_{5}, C_{6}\right) ; f_{1}=f_{1}\left(X, Y, Z ; x ; C_{5}, C_{6}\right)$;

$f_{2}=f_{2}\left(X, Y, Z ; x ; C_{5}, C_{6}\right) ; f_{3}=f_{3}\left(X, Y, Z ; x ; C_{5}, C_{6}\right)$. System equations, instead of this we obtain new system equations with non singular coeficients.

Now, if in case of nonsingular system equations for (2.1) proved that the system is the inteqrability, then we can find numerical or analytical solutions for nonsinular system equations of corrosponding systems (2.1).

\subsection{The Inteqrability of Nonlinear and Nonsingular System Equations (2.1)}

Now, we can rewrite the non-autonom system equations of (2.1) by account into from system (2.4) in the form

$$
\left\{\begin{array}{l}
\frac{\mathrm{d} X}{\mathrm{~d} x}=\frac{F_{1}(x, X, Y, Z)}{G_{1}(x, X, Y, Z)}, \\
\frac{\mathrm{d} Y}{\mathrm{~d} x}=\frac{F_{2}(x, X, Y, Z)}{G_{2}(x, X, Y, Z)}, \\
\frac{\mathrm{d} Z}{\mathrm{~d} x}=\frac{F_{3}(x, X, Y, Z)}{G_{3}(x, X, Y, Z)},
\end{array}\right.
$$

where, the functions $F_{i}(x, X, Y, Z), G_{i}(x, X, Y, Z), i=1,2,3$, dependence from the functions of $X, Y, Z$ and from space variable $X>0$, at the same time are analytical functions. From the theory of ODE and theories nonautonom ODE Prele-Singer and its modified extended procedure (Prele and Singer 1983; Duarte 
et al. 2002, and Lakshmanan M. and so on...) considered in case of nonsingular and nondegeneraing ODE systems in formally using from the classical theory of ordinary differential equations. These theories is also fit to the theory ODE. In our cases system ODE and its seting well-posed boundary conditions unknown. It is obviosly that general solution of the system (2.5) may be expressed by means of two constants as it form of first inteqrals view presentatitive forms. Suppose that this solution may be expressed in common form of $I(x, X, Y, Z)=C$, where constant $C$ must be selected in such form that, the total differential equtions there exists

$$
\mathrm{d} I=I_{X} \mathrm{~d} x+I_{X} \mathrm{~d} X+I_{Y} \mathrm{~d} Y+I_{Z} \mathrm{~d} Z=0,
$$

In this case using from the theory of ordinary differential equations the existance theorem of total ordinary differential equations, we can transforming Equation (2.5) in the equavalent form

$$
\left\{\begin{array}{l}
\frac{F_{1}(x, X, Y, Z)}{G_{1}(x, X, Y, Z)} \mathrm{d} x-\mathrm{d} X=0, \\
\frac{F_{2}(x, X, Y, Z)}{G_{2}(x, X, Y, Z)} \mathrm{d} x-\mathrm{d} Y=0, \\
\frac{F_{3}(x, X, Y, Z)}{G_{3}(x, X, Y, Z)} \mathrm{d} x-\mathrm{d} Z=0 .
\end{array}\right.
$$

As known from the courses of differential equations needed to find inteqrating factors suitable for each equation of the system (2.7). Let's denoting the inteqrating factors $R(x, X, Y, Z), K(x, X, Y, Z), M(x, X, Y, Z)$ respectively. Thus, multipling corresponding each of Equation (2.7) by $R(x, X, Y, Z)$, $K(x, X, Y, Z), M(x, X, Y, Z)$ respectively, we get

$$
R \frac{F_{1}}{G_{1}} \mathrm{~d} x-R \mathrm{~d} X=0, K \frac{F_{2}}{G_{2}} \mathrm{~d} x-K \mathrm{~d} Y=0, M \frac{F_{3}}{G_{3}} \mathrm{~d} x-M \mathrm{~d} Z=0,
$$

Hence, account the equality of (2.7) we have

$$
\mathrm{d} I=\left(R \varphi_{1}+K \varphi_{2}+M \varphi_{3}\right) \mathrm{d} x-R \mathrm{~d} X-K \mathrm{~d} Y-M \mathrm{~d} Z=0
$$

where, $\varphi_{i}=\frac{F_{i}(x, X, Y, Z)}{G_{i}(x, X, Y, Z)}, i=1,2,3$ or $\varphi_{1}=\frac{F_{1}(x, X, Y, Z)}{G_{1}(x, X, Y, Z)}=\frac{f_{1}(x)}{(f(x)+\varepsilon) 3 x^{3}}$, $\varphi_{2}=\frac{F_{2}(x, X, Y, Z)}{G_{2}(x, X, Y, Z)}=\frac{f_{2}(x)}{(f(x)+\varepsilon) 6 x^{3}}$, $\varphi_{3}=\frac{F_{3}(x, X, Y, Z)}{G_{3}(x, X, Y, Z)}=\frac{f_{3}(x)}{(f(x)+\varepsilon)(A(x)+\varepsilon) 6 x^{3}}$ are denoted. Comparsion the Equations (2.9). (2.7) with respect to $I_{X}, I_{X}, I_{Y}, I_{Z}$, gives

$$
I_{X}=R \varphi_{1}+K \varphi_{2}+M \varphi_{3}, I_{X}=-R, I_{Y}=-K, I_{Z}=-M
$$

Hence, in order there exists total differentiable from the equality of (2.7), needed to find the following main conditions for determine inteqrating factors (as usually for one equation $M(x, X)$ from classical theory must be satisfy necessary and suficiently conditions for total differential $\left.\mathrm{d} I(x, X)=I_{x} \mathrm{~d} x+I_{X} \mathrm{~d} X=0\right)$, but in our cases we would like take as $I(x, X, Y, Z)$, 
therefore nedded three function and between its codition of connection):

$R(x, X, Y, Z), K(x, X, Y, Z), M(x, X, Y, Z)$ in the forms

$$
\begin{gathered}
R_{X}+\varphi_{1} R_{X}+\varphi_{2} R_{Y}+\varphi_{3} R_{Z}=-\left(\varphi_{1 X} R+\varphi_{2 X} K+\varphi_{3 X} M\right) \\
K_{X}+\varphi K_{X}+\varphi_{2} K_{Y}+\varphi_{3} K_{Z}=-\left(\varphi_{1 Y} R+\varphi_{2 Y} K+\varphi_{3 Y} M\right) \\
M_{X}+\varphi_{1} M_{X}+\varphi_{2} M_{Y}+\varphi_{3} M_{Z}=-\left(\varphi_{1 Z} R+\varphi_{2 Z} K+\varphi_{3 Z} M\right) \\
R_{Y}=K_{X}, R_{Z}=M_{X}, R_{Y}=K_{X}, K_{Z}=M_{Y}
\end{gathered}
$$

Other sides, by inteqrating the equalities (2.9) account into the equalites (2.11) -(2.14), we get the following expression of motion inteqral formula

$$
I=r_{1}+r_{2}+r_{3}-\int\left[M+\frac{\mathrm{d}}{\mathrm{dZ}}\left(r_{1}+r_{2}+r_{3}\right)\right] \mathrm{d} z
$$

where, $r_{1}=\int\left[\varphi_{1} R+\varphi_{2} K+\varphi_{3} M\right] \mathrm{d} x, \quad r_{2}=-\int\left[R+\frac{\mathrm{d}\left(r_{1}\right)}{\mathrm{d} X}\right] \mathrm{d} X$, $r_{3}=-\int\left[K+\frac{\mathrm{d}\left(r_{1}+r_{2}\right)}{\mathrm{d} Y}\right] \mathrm{d} Y$.

Or

$$
r_{1}=\int\left[R \frac{f_{1}(x)}{(f(x)+\varepsilon) 3 x^{3}}+K \frac{f_{2}(x)}{(f(x)+\varepsilon) 6 x^{3}}+M \frac{f_{3}(x)}{(f(x)+\varepsilon)(A(x)+\varepsilon) 6 x^{3}}\right] \mathrm{d} x,
$$

$$
r_{2}=-\int\left[R+\frac{\mathrm{d}}{\mathrm{d} X}\left(\int\left[R \frac{f_{1}(x)}{(f(x)+\varepsilon) 3 x^{3}}+K \frac{f_{2}(x)}{(f(x)+\varepsilon) 6 x^{3}}+M \frac{f_{3}(x)}{(f(x)+\varepsilon)(A(x)+\varepsilon) 6 x^{3}}\right] \mathrm{d} x\right)\right] \mathrm{d} X,
$$$$
r_{3}=-\int\left[K+\frac{\mathrm{d}}{\mathrm{d} Y}\left[\int\left[R \frac{f_{1}(x)}{(f(x)+\varepsilon) 3 x^{3}}+K \frac{f_{2}(x)}{(f(x)+\varepsilon) 6 x^{3}}+M \frac{f_{3}(x)}{(f(x)+\varepsilon)(A(x)+\varepsilon) 6 x^{3}}\right] \mathrm{d} x\right.\right.
$$

$$
\left.-\int\left[R+\frac{\mathrm{d}}{\mathrm{d} X}\left(\int\left[R \frac{f_{1}(x)}{(f(x)+\varepsilon) 3 x^{3}}+K \frac{f_{2}(x)}{(f(x)+\varepsilon) 6 x^{3}}+M \frac{f_{3}(x)}{(f(x)+\varepsilon)(A(x)+\varepsilon) 6 x^{3}}\right] \mathrm{d} x\right)\right] \mathrm{d} X\right] \mathrm{d} Y
$$

Let the functions $S(X, Y, Z, X), U(X, Y, Z, X)$ are such that the equality $R$ $=S M, K=U M$ be satisfied. Then using the operator $D=\frac{\partial}{\partial x}+\varphi_{1} \frac{\partial}{\partial X}+\varphi_{2} \frac{\partial}{\partial Y}+\varphi_{3} \frac{\partial}{\partial Z}$, we get the following equalities

$$
\begin{gathered}
D(S)=S\left[\left(S \varphi_{1 Z}+U \varphi_{2 Z}+\varphi_{3 Z}\right)-\left(S \varphi_{1 X}+U \varphi_{2 X}+\varphi_{3 X}\right)\right], \\
D(U)=U\left[\left(S \varphi_{1 Z}+U \varphi_{2 Z}+\varphi_{3 Z}\right)-\left(S \varphi_{1 Y}+U \varphi_{2 Y}+\varphi_{3 Y}\right)\right], \\
D(M)=M\left[S \varphi_{1 Z}+U \varphi_{2 Z}+\varphi_{3 Z}\right], \\
M_{X}=S M_{Z}+M S_{Z}, M_{Y}=U M_{Z}+M U_{Z}, U_{X}-S_{Y}=S U_{Z}-U S_{Z}
\end{gathered}
$$

Remark 21. Instead of $R=S M, K=U M$ we can take $R=\bar{S} K, M=\bar{U} K$ or $K=\bar{S} R, M=\bar{U} R$ then for $I_{\chi}, I_{X}, I_{Y}, I_{Z}$ first integrals we get five possiblity classes catagoris:

1) $I_{X}=0, I_{X} \neq 0, I_{Y} \neq 0, I_{Z} \neq 0$;

2) $I_{Y}=0, I_{X} \neq 0, I_{X} \neq 0, I_{Z} \neq 0$; 
3) $I_{X}=0, I_{Z} \neq 0, I_{X} \neq 0, I_{Y} \neq 0$;

4) $I_{X} \neq 0, I_{X} \neq 0, I_{Y} \neq 0, I_{Z} \neq 0$;

5) $I_{X} \neq 0, I_{X} \neq 0, I_{Y} \neq 0, I_{Z} \neq 0$.

In order to find the conditions of existances first inteqrals for example, in case of $I_{X}=0, I_{X} \neq 0, I_{Y} \neq 0, I_{Z} \neq 0$. In this case since $I_{x}=0$ then $R=0$. Hence, there are the following possible conditions: 1) $S=0$ and $M \neq 0,2) M=0$ and $S \neq 0$ are established. Thus, we have: 1) $S=0$ and $M \neq 0,2) M=0$ and $S \neq 0$, hence, we find the function $U$ in the form $U=-\frac{\varphi_{3 X}}{\varphi_{2 X}}, U_{X}=0$. Now we can find the function $M$ from the following equations:

$$
\begin{aligned}
& \left.\quad D(M)=M\left[S \varphi_{1 Z}+U \varphi_{2 Z}+\varphi_{3 Z}\right], U=-\frac{\varphi_{3 X}}{\varphi_{2 X}}, U_{X}=0\right), S=0 \text { and } M \neq 0, \\
& M_{X}=S M_{Z}+M S_{Z}, \quad M_{Y}=U M_{Z}+M U_{Z}, U_{X}-S_{Y}=S U_{Z}-U S_{Z}, \quad R=S M=0 \\
& \text { and } K=U M, \quad K=-\frac{\varphi_{3 X}}{\varphi_{2 X}} M, M_{X}=0, M_{Y}=-\frac{\varphi_{3 X}}{\varphi_{2 X}} M_{Z}+M\left[\frac{\varphi_{3 X}}{\varphi_{2 X Z}}\right]_{Z}=0, \\
& M_{Z}=-\frac{\varphi_{2 X}}{\varphi_{3 X}} M\left[-\frac{\varphi_{3 X}}{\varphi_{2 X Z}}\right]_{Z}
\end{aligned}
$$

From the equalities $K=U M, R=S M$ we can find by the values of $R(x, X, Y, Z), K(x, X, Y, Z)$, the $M(x, X, Y, Z)$. Thus account into the integrals $r_{1}, r_{2}, r_{3}$ in the first motion inteqral $I=I(x, X, Y, Z)=C_{\rightarrow}$, finally we get the following expression: $I=r_{1}+r_{2}+r_{3}-\int\left[M+\frac{\mathrm{d}}{\mathrm{d} Z}\left(r_{1}+r_{2}+r_{3}\right)\right] \mathrm{d} z$. Hence, we can able to formulate the theorem of inteqrability, it means that the system nonsingular equations is solvability:

Theorem 2.1 (inteqrabilty of (2.5)). Let the conditions $R_{Y}=K_{X}, R_{Z}=M_{X}$, $R_{Y}=K_{X}, K_{Z}=M_{Y}$ are satisfied. Then, in order there existence the first integral of system (2.1) (or (2.3)), it is sufficiently must be satisfy the equality: $\varphi_{3 X}\left(\varphi_{2 X X}+\varphi_{2} \varphi_{2 X Y}+\varphi_{3} \varphi_{2 X Z}-\varphi_{2 Z} \varphi_{3 X}-\varphi_{2 X} \varphi_{2 Y}\right)$ $-\varphi_{2 x}\left(\varphi_{3 X X}+\varphi_{2} \varphi_{3 X Y}+\varphi_{3} \varphi_{3 X Z}-\varphi_{2 X} \varphi_{3 y}-\varphi_{3 x} \varphi_{2 Z}\right)=0$. Thus, we obtain that the nonlinear and non autonom ordinary system Equations (2.5) (or (2.4)), which is corresponding to system equations of (2.1)-(2.3) without initial conditions is integrability. Now we can able to begin finding analitycal (or numerical) solution.

Remark 3. Identically, similarly way the following orthers cases can be investigated from the conditions

2) $I_{Y}=0, I_{X} \neq 0, I_{X} \neq 0, I_{Z} \neq 0$;

3) $I_{X}=0, I_{Z} \neq 0, I_{X} \neq 0, I_{Y} \neq 0$;

4) $I_{X} \neq 0, I_{X} \neq 0, I_{Y} \neq 0, I_{Z} \neq 0$;

5) $I_{X} \neq 0, I_{X} \neq 0, I_{Y} \neq 0, I_{Z} \neq 0$.

As it shown in the work [7] [8] [9] [10] [11], the theory of boundary value problems for degenerate equations it is a well-known fact that the well-posedness and the class of its correctness essentially depend on the coefficients. Great difficulties come into being in the investigation of systems of degenerating equations. Therefore, in spite of proved the integrality of system equations in formally, let's additionally, to attempt prove the solvability of system equations of (2.1) by means of system of regularizing equations of (2.3) (or (2.4)) in weighted 
spaces. For this reason we must to choose suitable spaces which are corresponding to well-posed boundary conditions. Thus by the symbol $\mathrm{C}_{\mathrm{L}}$ we denote a class of continuously differentiable functions in the domain $D=\{x>0\} \cap\left\{c^{*}>0.86\right\}$, satisfying the initial conditions $X\left(c^{*}\right)=2, Y\left(c^{*}\right)=5 X\left(c^{*}\right), Z\left(c^{*}\right)=1$, where $c^{*}=1$ which is also taken by Parker's (where $Z\left(c^{*}\right)$ is observation value). Denoting by the space $H_{1}(D)$ in the Sobolev's space (see [12]) with weighted spaces obtained for $C_{L}$ class functions (i.e. $X(x), Y(x), Z(x) \in C_{L}$ ) which is closed by the norm: $\|U(x)\|_{H_{1}(D)}^{2}=\int_{D}\left[\sqrt{f(x)+\varepsilon}\left(\frac{\mathrm{d} U}{\mathrm{~d} x}\right)^{2}+U^{2}(x)\right] \mathrm{d} x$. Since $f(x) \neq 0$ for $x=c^{*} \neq c$-critical point then $X(x), Y(x), Z(x) \in H_{1}(D) \cap W_{2}^{1}\left\{D \backslash\left\{x=c^{*}=c\right\}\right\}$ and satisfy initial conditions.

Definition 2.2. The function $U(x)$ is said to be generalized solution, if $U(x) \in H_{1}(D) \cap L_{2}(D)$ be satisfied the equality $\left(U_{\varepsilon}(x), \varphi(x)\right)_{L_{2}(D)}=(-F(x), \varphi(x))_{L_{2}(D)}$, where $\varphi(x) \in C_{0}^{1}(D)$ is set of test functions (i.e.supp $\varphi(x) \in C^{\prime}(D \backslash$ boundary $)$.

Theorem 2.2. Let the $\lambda<0$ is sufficiently large number, then for continuous function $F(x)$ the inequality is holds true:

$$
\left\|U_{\varepsilon}(x)\right\|_{H_{1}(D)} \leq m\|F(x)\|_{L_{2}(D)} .
$$

Proof. Indeed, multiplying the Equation (2.3), by $\mathrm{e}^{\lambda x} U_{x}^{\prime}(x)\left(\mathrm{e}^{\lambda x} \frac{\mathrm{d} U}{\mathrm{~d} x}\right)$ and after integrating by parts, account into initial conditions, in additionally, also using inequality of Cauchy-Bunyakovski, we get this priory estimate. Hence, include that the functions of family $U_{\varepsilon}(x)$ are uniformly bounded, then in equality $\left(U_{\varepsilon}(x), \varphi(x)\right)_{L_{2}(D)}=(-F(x), \varphi(x))_{L_{2}(D)}$, we may pass to limit in standard form transition procedure for weak solution in the integral equality (see [7] [8] [9]), when $\varepsilon \rightarrow 0$.

Definition 2.3. The functions $U_{\varepsilon}(x) \in H_{1}(D) \cap W_{2}^{1}(D)$ is said to be a regular solution of considered problem (system equation and its boundary conditions), if it is generalized solution which is satisfy almost everywhere system equations of (2.1). Since the inequality (2.20) is hold true, then there exists trace of $U_{\varepsilon}(x) \in H_{1}(D) \cap W_{2}^{1}\left\{x \cap c^{*}, c^{*} \neq c\right\}$. Other side, the function $F(x)$ is differentiable, then for $U_{n}(x) \in C_{L}(D)$ sequences functions the following is holds true: $\lim _{n \rightarrow \infty}\left\|\sqrt{f(x)+\varepsilon} \frac{\mathrm{d} U_{n}(x)}{\mathrm{d} x}-F(x)\right\|_{L_{2}(D)}=\lim _{n \rightarrow \infty}\left\|U_{n}(x)-U(x)\right\|_{H_{1}(D)}=0$.

Hence, we may include that there exists regular solution and by the theory of strong solution it is identity with weak solution (see [7] [8] [9] [11] [13]). Thus, we can include that, the solution $U(x) \in H_{1}(D)$ is gluing solutions of $U^{-}(x)=U^{+}(x)$ at the point of critical which this point transition as supersonic lines. This theorem show that regularizing solutions of system Equations (2.3) (or (2.4)) almost identity with solutions of system equations of (2.1). Therefore, we may able to find (by strictly satisfying law of basic transition rule) solution of system (2.1) by analytical and numerical forms is held simultaneously. Now, on 
the base of the fundamentals base of established ours theory we can seek solution of given problem in view of complex couple solution of analytically formulas and numerically cases.

\section{Analytical and Numerical Presentation of Solution of the System Equations (2.1)}

Since, $f(x)=\frac{1}{2} A(A-4)+\frac{4 B}{3 X}, f(x)=0$ is natural singularity coefficient, we must to use directly it, other sides, $A(x)=0$ degenerating line (sonic line), therfore from the fixed point and by the theory of free boundary conditions (see [7] [8] [9] [10] [11] and theirin) there exests $x=c^{*}$ point and chosing from $(A+\varepsilon)$ such that must be satisfy $f\left(c^{*}\right)=0$. Hence instead of $\varepsilon>0$ choosing any number we may establish from the $f\left(c^{*}\right)=0$ no degenerating case, but at the same time establishing free boundary value conditions for functions $X\left(c^{*}\right)$, $Y\left(c^{*}\right)$ using by observation value of $Z\left(c^{*}\right)$. Hence, we have $\frac{4 B\left(c^{*}\right)}{3 X\left(c^{*}\right)}=2$ or $2 B\left(c^{*}\right)=3 X\left(c^{*}\right)$. Hence, for comfortable chosen $B\left(c^{*}\right)=3$, $X\left(c^{*}\right)=2$, As it chosen by Parker's [1] fixed point $c^{*}=1$, and therefore $Z\left(c^{*}\right)=Z(1)=1$, consequently, we have $X(1)=2$ and from the $A(x)=0$ obtained $Y(1)=2$ fixed boundary conditions is established. Now, we able to establish classical well-posed boundary value problem in natural law of theory differential equations. This theory belongs to the so-called non-classical theory of ODU (or PDE), at the same time this model approaches satisfy definition of "Non-classical_Definition of Boundary Value Problem". Using by common factors between of $B\left(c^{*}\right), X\left(c^{*}\right)$, i.e. $2 B\left(c^{*}\right)=3 X\left(c^{*}\right)$ we chose the $k$ number and using $Z\left(c^{*}\right)=Z(1)=1, Z(c)=C$ observation value at the point $x=c$ the feture constructing solution $X(x), Y(x), Z(x)$ will be analytical, because by this method we provide the continuty of these fuctions. Note that we obtain three analytical solutions and at the any points of domain of $x>0$ admissable the continuty values of these $X(x), Y(x), Z(x)$. Finally, account into above, the system (2.1) which is after derivation to nonautonom system of ODE is being to nonsingular and nondegeneraiting ordinary system equations first order in the following form:

$$
\begin{aligned}
& \frac{\mathrm{d} X}{\mathrm{~d} x}=\frac{-4 Z(x)}{x^{3}}+\frac{5}{3 x^{2}}+\frac{1}{x^{3}}, \frac{\mathrm{d} Y}{\mathrm{~d} x}=\frac{-6 Z(x)}{x^{3}}+\frac{7}{3 x^{2}}+\frac{2}{x^{3}}, \\
& \frac{\mathrm{d} Z}{\mathrm{~d} x}=-\frac{1}{2 x^{3} X(x)}\left[Z^{2}(x)-\frac{7 x}{6}-\frac{5}{6} x Z(x)+\frac{5}{4} x+\frac{3}{4}\right],
\end{aligned}
$$

Substituting constants $C_{5}=7, C_{6}=1,5$, we have analytical solutions respectto $X(x), Y(x), Z(x)$ in the following (which is the constant $C_{1}$ is arbitrarily and allowance to use boundary conditions for family of solutions):

$$
\begin{aligned}
& X(x)=\frac{1}{21} \frac{1}{x}-\frac{1}{14 x^{2}}+C_{1}\left(-\frac{1}{7}\right), Y(x)=5 X(x)=\frac{5}{21} \frac{1}{x}-\frac{5}{14 x^{2}}+C_{1}\left(-\frac{5}{7}\right), \\
& Z(x)=\frac{3}{7} x+\frac{3}{14}
\end{aligned}
$$


Remark 3.1. Starting from the formula $2 B\left(c^{*}\right)=3 X\left(c^{*}\right)$ for each point of domain $[1, \infty) \cap\left(c^{*}>c\right.$-critical $)$ accounting into the numbers $k \in N$ as the common factor between of 3 and 2 for all points of admissable variables $X$, the boundary conditions can be established (sometimes these initial value condtions is so-called as moiving boundary conditions) for system equations of (2.1). In this case these moiving boundary conditions allowance to determine all solutions for $x>c$-critical points which is the functions $X, Y, Z$ representatives by analitical formulas having sketch graph as is it shown in Figure 1(a) and Figure 1(b).

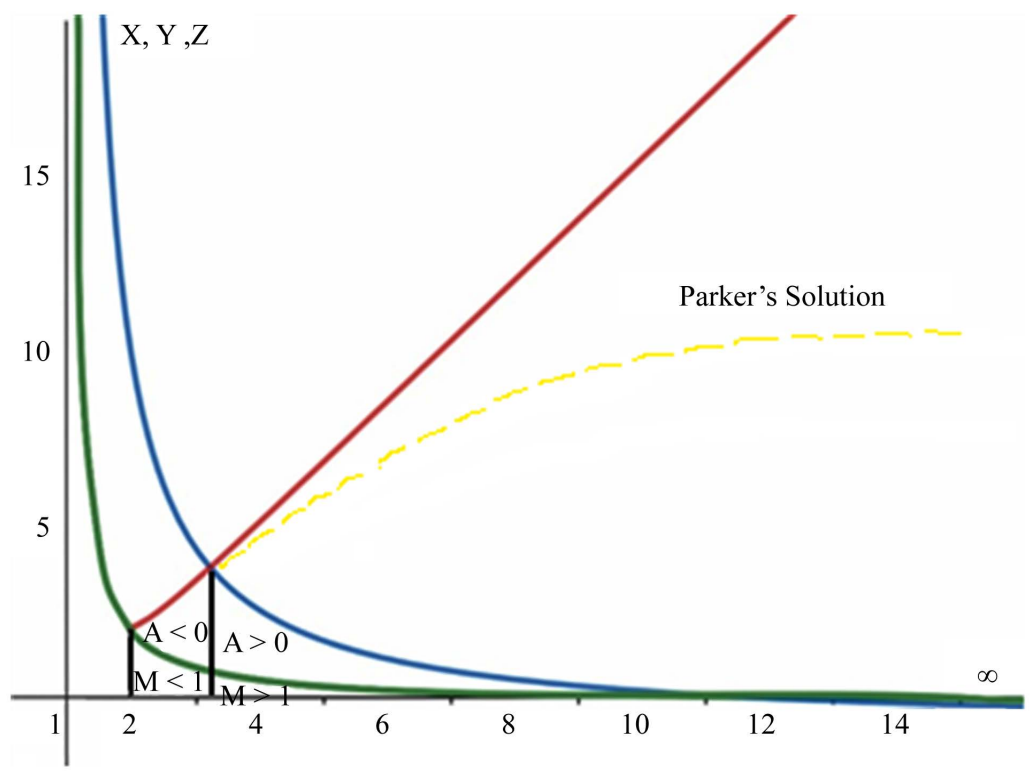

(a)

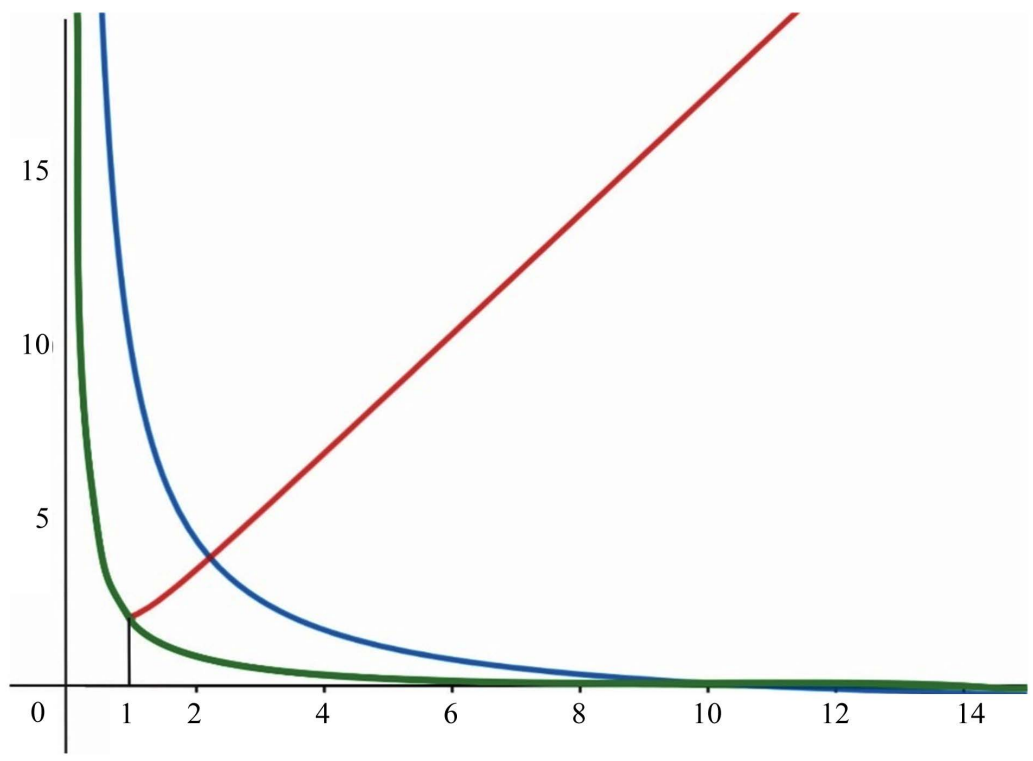

(b)

Figure 1. (a) The graph of solutions family which included Parker's solutions; (b) The graph of solutions family. 


\section{Conclusions}

Our derived Equations (2.1) are a generalization of the Parker problem to the case of anisotropic radial and stationary solar wind. These equations are new and have not been investigated by anyone. The main difficulty in solving these equations is related to the zeros of the functions $f(x)$ and $A(x)$ in the region of integration $1 \leq x \leq \infty$. However, the equations contain two unknown arbitrary constants $C_{5}, C_{6}$ which make the singularities avoidable. One of the great difficulties is the problem of finding these constants. After this it is necessary to set the initial boundary conditions and simultaneously it is necessary to restore the nonsingularity and nondegeneracy of the system of equations. After these hurdles difficulties only come non-autonomous and nonlinear system of equation, which also its turn is difficultly solvable. And so after established above mentioned steps of difficulties the following results are obtained:

1) First of all no lost the singularity and degenerating cases we find value of the constant $C_{5}, C_{\dot{\sigma}}$

2) Constructed by nonclassical approches the initial conditions which include movable boundary conditions;

3) Using nonclassical methods, with aid " $\varepsilon$-regularization" and "fixed point", priory estimations established theorems of solvability for given non-autonomous, nonlinear system of ordinary differential equations under consideration, when the coefficients degenerate, simultaneously having singularities;

4) After constructed nonsingular system equations established well-posed boundary value problem, founded the analytical solution;

5) Using a especially numerical method obtained the classes solution which is applying so-called "Hybrid" (complex couple form of analytical-numerical method simultaneously) established in spaces $\left(x \cap c^{*}\right.$ (where, $\left.c^{*}>0.86\right)$;

6) All solutions, which is corresponding definition of domain as shown by Parker's (we prove that must be $c^{*}>0.86$ );

7) Using program, established sketch of graph of these solution families as shown in Figure 1(a) and Figure 1(b).

\section{Acknowledgements}

This work was supported by the Science Development Foundation under the President of the Republic of Azerbaijan-Grant No. EİF-BGM-4-RFTF-1/2017-21/06/1.

\section{Conflicts of Interest}

The authors declare no conflicts of interest regarding the publication of this paper.

\section{References}

[1] Parker, E.N. (1958) Dynamics of the Interplanetary Gas and Magnetic Fields. The Astrophysical Journal, 128, 664-676. https://doi.org/10.1086/146579

[2] Weber, E.J. and Davis Jr., L. (1967) The Angular Momentum of the Solar Wind. 
The Astrophysical Journal, 148, 217-227. https://doi.org/10.1086/149138

[3] Baranov, V.B. and Krasnobayev, K.V. (1977) Hydrodynamics of Cosmic Plasma. Nauka, Moscow, 335.

[4] Dzhalilov, N.S., Kuznetsov, V.D. and Staude, J. (2008) Wave Instabilities in an Anisotropic Magnetized Space Plasma. Astronomy Astrophysics, 489, 769-772. https://doi.org/10.1051/0004-6361:200809882

[5] Kuznetsov, V.D. and Dzhalilov, N.S. (2009) Sixteen-Moment Approximation for a Collisionless Space Plasma: Waves and Instabilities. Plasma Physics Reports, 35, 962-975. https://doi.org/10.1134/S1063780X09110063

[6] Dzhalilov, N.S., Aliev, N.A. and Ismailov, N.A. (2014) On the Partial Solution of the Equation of the Anisotropic Solar Wind. Institute of Applied Mathematics, 3, 3-14.

[7] Nurmamedov, M.A. (2015) The Existence and Uniqueness of a New Boundary Value Problem (Type of Problem "E”) for Linear System Equations of the Mixed Hyperbolic Elliptic Type in the Multivariate Dimension with the Changing Time Direction. Abstract and Applied Analysis, 2015, Article ID 7036552. https://doi.org/10.1155/2015/703652

[8] Nurmamedov, M.A. (2015) The Solvability of a New Boundary Value Problem with Derivatives on the Boundary Conditions for Forward-Backward Linear Systems Mixed of Keldysh Type in Multivariate Dimension Sciences Publishing. International Journal of Theoretical and Applied Mathematics, 1, 1-9.

[9] Nurmamedov, M.A. (2015) The Solvability of a New Boundary Value Problem with Derivatives on the Boundary Conditions for Forward-Backward Semi Linear Systems of Mixed Equations of Keldysh Type in Multivariate Dimension. Sciences Publishing. International Journal of Theoretical and Applied Mathematics, 1, 10-20.

[10] Keldysh, M.V. (1951) On Some Cases of Degenerate Elliptic Equations on the Boundary of a Domain, Doklady Acad. Proceedings of the USSR Academy of Sciences, 77, 181-183.

[11] Nurmamedov, M.A. (1985) The First Boundary Value Problems for One Model Equations of Mixed Type-Nonclassical Equations of Mathematical Physics. Proceeding of Institute of Mathematics of Siberian Branch of the Academy of Sciences USSR, Novosibirsk, 117-122.

[12] Sobolev, S.L. (1963) Applications of Functional Analysis in Mathematical Physics, Izdat. Translations of Mathematical Monographs, Leningrad.

https://doi.org/10.1090/mmono/007

[13] Sarason, L. (1962) On Weak and Strong Solutions of Boundary Value Problems. Communications on Pure and Applied Mathematics. 15, 237-288.

https://doi.org/10.1002/cpa.3160150301 